\title{
Optimizing WiMAX: Mitigating Co-Channel Interference for Maximum Spectral Efficiency
}

\author{
ABDUL QADIR ANSARI*, ABDUL LATEEF MEMON**, AND IMRAN ALI QURESHI** \\ RECEIVED ON 14.03.2016 ACCEPTED ON 11.05.2016 \\ ABSTRACT
}

\begin{abstract}
The efficient use of radio spectrum is one of the most important issues in wireless networks because spectrum is generally limited and wireless environment is constrained to channel interference. To cope up and for increased usefulness of radio spectrum wireless networks use frequency reuse technique. The frequency reuse technique allows the use of same frequency band in different cells of same network considering inter-cell distance and resulting interference level. WiMAX (Worldwide Interoperability for Microwave Access) PHY profile is designed to use FRF (Frequency Reuse Factor) of one. When FRF of one is used it results in an improved spectral efficacy but also results in CCI (Co-Channel interference) at cell boundaries. The effect of interference is always required to be measured so that some averaging/ minimization techniques may be incorporated to keep the interference level up to some acceptable threshold in wireless environment.
\end{abstract}

In this paper, we have analyzed, that how effectively CCI impact can be mitigated by using different subcarrier permutation types presented in IEEE 802.16 standard. A simulation based analysis is presented wherein impact of using same and different permutation base in adjacent cells in a WiMAX network on CCI, under varying load conditions is analyzed. We have further studied the effect of permutation base in environment where frequency reuse technique is used in conjunction with cell sectoring for better utilization of radio spectrum.

Key Words: Worldwide Interoperability for Microwave Access, Co-Channel Interference, FRF-Frequency Reuse Factor, Cell Sectoring, Orthogonal Frequency Division Multiplexing, Orthogonal Frequency Multiple Access.

\section{INTRODUCTION}

W IMAX has been projected to provide wired equivalent quality for wireless data services and is targeted to provide competitive service profile for wireless application well comparable to wired broadband access technologies such as DSL (Digital Subscriber Line) [1]. The IEEE 802.16e air interface was spruced up to support mobile environment and to defy varying channel conditions. The IEEE 802.16e-2005 standard include different PHY layer specifications; SC (Single-Carrier), OFDM

* Department of Biomedical Engineering, Mehran University of Engineering \& Technology, Jamshoro.

** Department of Telecommunication Engineering, Mehran University of Engineering \& Technology, Jamshoro.

Mehran University Research Journal of Engineering \& Technology, Volume 35, No. 4, October, 2016 [p-ISSN: 0254-7821, e-ISSN: 2413-7219] 
(Orthogonal Frequency Division Multiplexing), and OFDMA (Orthogonal Frequency Division Multiple Access). TDMA (Time Division Multiple Access) is at core of SC and OFDM; however OFDMA uses both the time and frequency for channel resource allocation. WiMAX Forum declared OFDMA [2] as the basic multiple access technology.

\section{ORTHOGONAL FREQUENCY DIVISION MULTIPLE ACCESS}

Broadband wireless access requires high order modulation schemes with better spectral efficiency to enable wired equivalent transfer rate/connectivity and quality. In comparison to widely used analog modulation techniques such as AM (Amplitude Modulation), FM (Frequency Modulation) and less efficient digital modulation techniques such as PSK (Phase Shift Keying), BPSK (Binary PSK), and QPSK (Quadrature PSK) schemes used in wireless access are sensitive to interference due to typical nature of wireless signal and the basic phenomenon of multi-path propagation in wireless transmission. This goes even more difficult in broadband wireless networks where fundamental premise is high data rate, ubiquity with large numbers of users.

OFDM assigns all Sub-Carriers of a symbol to one specific user; however OFDMA allows assignment of subcarriers in a symbol to multiple users. OFDM distributes the signal into various narrow-band signals also called sub-channels or subcarriers of different frequencies $[3,4]$ and a subscriber is assigned one or more sub-channels for transmission. In OFDMA allocation of transmission resources is based on OFDM symbol and sub channels; thus providing high granularity bandwidth allocation using both time and frequency multiple access [5,6]. Data Subcarriers, Pilot Subcarrier and Null Subcarrier are three subcarrier types defined with subcarrier spacing of 10.9375 $\mathrm{kHz}[5]$.
The IEEE-802.16e-2005 PHY standard offers two different schemes for sub-channel allocation [7].

(a) Distributed Subcarrier Permutation: In distributed subcarrier permutation a sub-channel uses subcarriers randomly distributed across the channel bandwidth. There are again two variants of distributed cub-carrier scheme (Fig. 1).

(a) Subcarriers are Distributed over the Entire Frequency of Channel. This is stated to as FUSC (Fully Utilized-Sub-Channelization).

(b) Some Distributed Clusters of Subcarriers Constitute a Sub-Channel. This is stated to as PUSC (Partially-Used-Sub-Channelization).

(b) Contiguous Subcarrier Permutation: In contiguous subcarrier permutation type, a subchannel uses neighboring subcarriers that are carefully chosen by the scheduler (Fig. 1). This is called AMC (Adaptive Modulation and Coding) which is very useful in meeting high bandwidth requirements often constrained due varying channel conditions in wireless transmission.

\section{DISTRIBUTED SUBCARRIER PERMUTATION}

\subsection{Fully-Utilized-Sub-Channelization}

In FUSC all of the SCs (Sub Channels) are allocated to the transmitter. That is why it is called fully utilized sub channel. FUSC can be used in DL only [3]. One slot of FUSC DL is one OFDM symbol by one SC and one FUSC DL SC contains 48 data-subcarriers. Using 2048-FFT, total number of SC is 32. From a total of 2048 subcarriers, 1536 are data subcarriers, 173 Left-Guard, 172 Right-Guard, 166 Pilot subcarriers (24 fixed Pilots, 142 Pilot subcarriers are

Mehran University Research Journal of Engineering \& Technology, Volume 35, No. 4, October, 2016 [p-ISSN: 0254-7821, e-ISSN: 2413-7219] 
variable i.e. change from symbol to another) and one DC subcarriers. Data subcarriers form a FUSC group, using 2048 FFT, there could be 48 FUSC groups and 32 subcarriers constitute one FUSC group.

\subsubsection{Sub-Channel Formation in FUSC}

Prior to assignment of subcarriers to SC, pilot subcarriers are divided into fixed and variable type. FUSC groups are formed from data subcarriers and then after permutation is applied using equation 1 to allocate subcarriers to SC [3]. In a sub-channel there could be 48 data subcarriers; also it is important to note that number of SC is equal to subcarriers in a FUSC group alternatively it may be stated that using 2048-FFT, there could be 32 Sub-channels in total.

During the permutation process of subcarrier distribution, one subcarrier is selected from every FUSC group and is being allocated to a SC. The Equation (1) is permutation formula for allocation of subcarriers from FUSC group to SC.

Subcarrier $(\mathrm{k}, \mathrm{s})=\mathrm{N}_{\text {subchannels }} \cdot \mathrm{n}_{\mathrm{k}}+\left\{\mathrm{P}_{\mathrm{s}}\left[\mathrm{n}_{\mathrm{k}} \bmod \mathrm{N}_{\text {subchannels }}\right]+\right.$

DL_PermBase $\}$ mod $\mathrm{N}_{\text {subchannels }}$

\subsection{Partial Usage of Sub-Channels}

In PUSC sub-channels are distributed among different transmitters. That is why it is called partial usage of SC. The SCs allotted to one transmitter make one segment. Left behind sub-channels are ascribed to other different segments and thus to different sectors.

Two OFDM symbols by one SC for one PUSC DL slot; and 24 data subcarriers form one PUSC DL.

From a total of 2048 subcarriers, 1440 are data subcarriers, 184 Left-Guard, 183 Right-Guard, 240 Pilot subcarriers and one DC subcarrier. Inner permutation of logical clusters
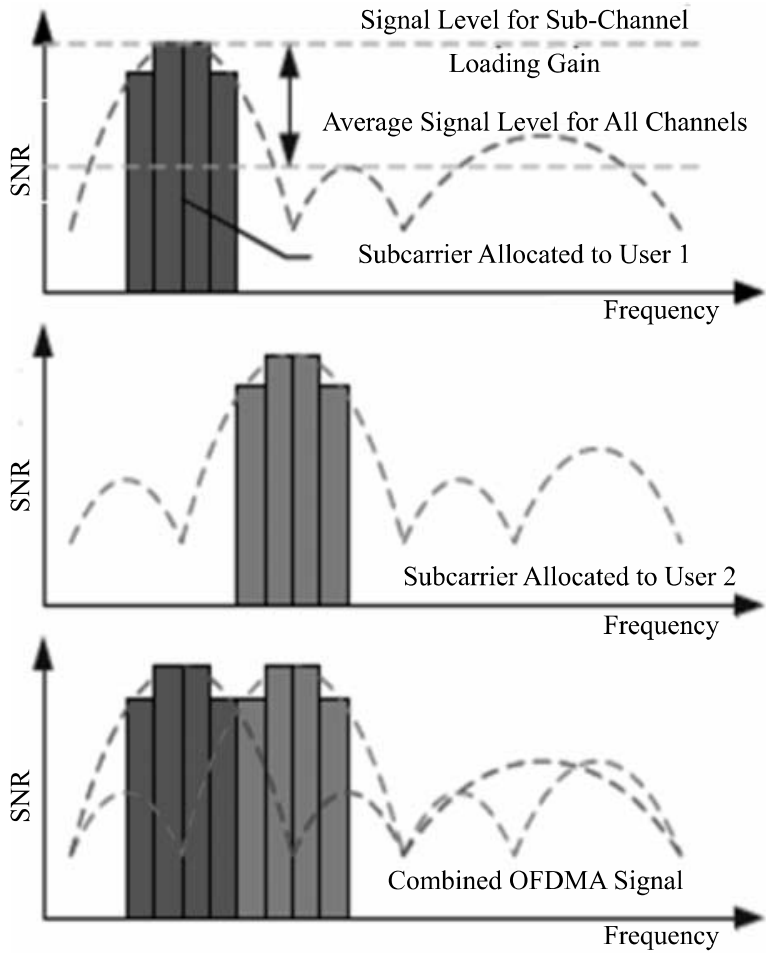

Contiguous
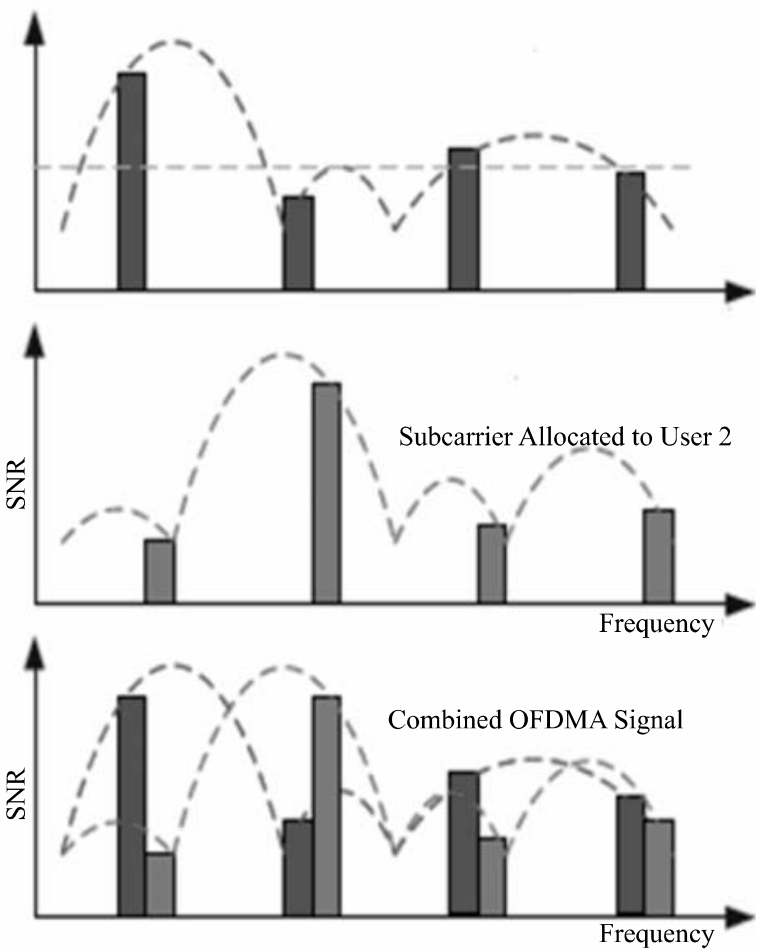

Distributed

FIG. 1. CONTIGUOUS AND DISTRIBUTED SUBCARRIER PERMUTATION [7] 
of a group is carried to make SCs from the subcarrier. Permutation base may be selected from 0-31 for the rest of PUSC zones (consist of OFDMA symbols with same permutation scheme).

\subsubsection{Sub-Channel Formation in PUSC}

The permutation process to form Sub-Channels is shown in Fig. 2. There are two intrinsic processes called Inner Permutation and Outer Permutation (Fig. 3). The Inner Permutation is used to make Sub-Channels from subcarriers in logical clusters of a group. As already stated Permutation base may be chosen from 0-31 for remaining
PUSC zones. Then Physical clusters are renumbered to logical clusters using process of Outer-Permutation.

In DL PUSC available subcarriers are distributed as data, pilot, DC, right guard band and left guard band subcarriers. In 2048-FFT, there are total 120 physical clusters (14 contiguous subcarriers form a physical cluster). These clusters include all the data and pilot subcarriers. Physical clusters are renumbered using Equation (2) to form logical clusters (Fig. 4).

Logical_Clusture $=$ Renumbering_Sequence

(Phycial $_{\text {Clusture }}+13 *$ DL Permbase

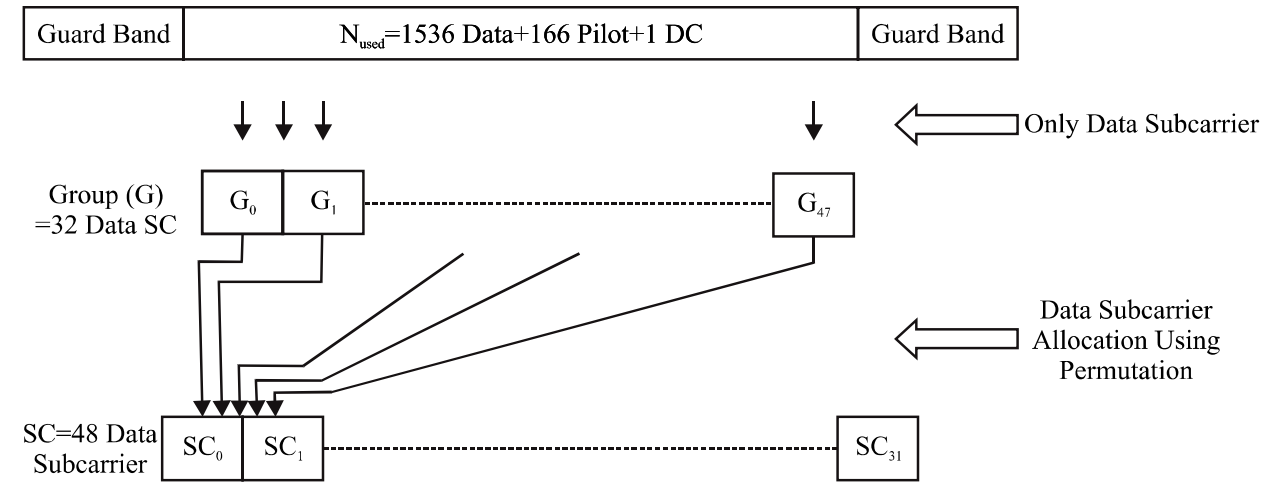

FIG. 2. PERMUTATION PROCESS OF FUSC DOWNLINK [3].

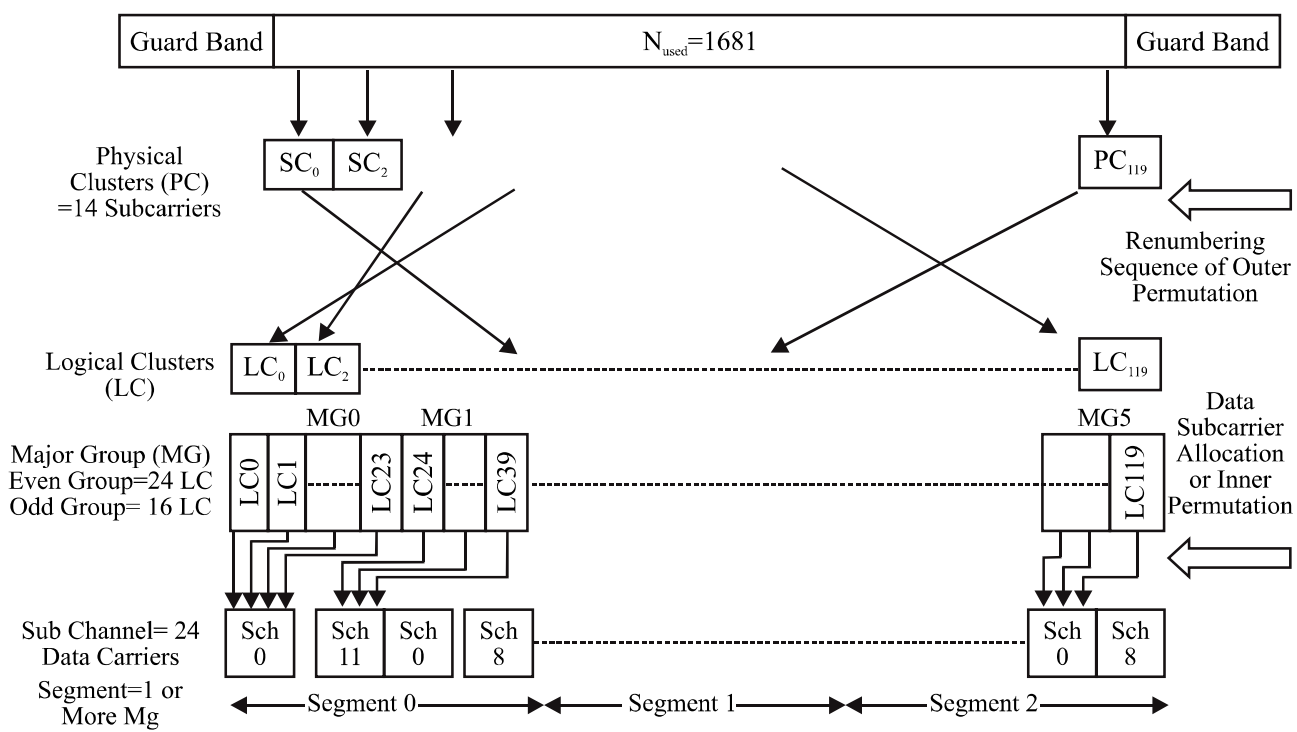

FIG. 3. OUTER AND INNER PERMUTATIONS IN PUSC DL [3] 
These logical clusters are distributed in 6 groups (numbered $0-5)$ such that even $(0,2$, and 4$)$ groups have 24 clusters each and odd (1, 3, and 5) groups have 16 clusters each. This is how this outer permutation gives frequency diversity. Separate marking of Pilot positions is required before inner permutation. For instance logical cluster 2 is the $37^{\text {th }}$ physical cluster (refer equation 3 for logical cluster set and the subcarriers are from $0-13$. The logical positions pertaining to the absolute subcarrier index $(0-2047)$ is 702-715. For odd symbols the pilot subcarriers will be 4 and 8 (i.e. 706 and 710 pertaining to absolute subcarrier index); similarly pilot subcarriers for even OFDM symbols will be 0 and 12 (i.e. 702 and 713 pertaining to absolute subcarrier index). The inner permutation will utilize remaining 12 subcarriers.
Subcarriers of a group are assigned to sub-channels using permutation formula as given in Equation (1).

In uplink PUSC one slot of PUSC UL is three OFDM symbols by one sub-channel. From a total of 2048 subcarriers, there are 184 Left-Guard, 183 Right-Guard subcarriers and one DC subcarrier. For even numbered OFDM symbol there are 840 data subcarriers and same number of pilot subcarriers i.e. 840 pilots. However for Odd numbered symbols there are 1680 data and no pilot. UL PUSC Sub-channel is formed from six logical tiles (Fig. 5).

The permutation process to map data points on subcarriers of sub-channels is such that Physical tiles are renumbered as logical tiles using Equation (3) (Fig. 6).

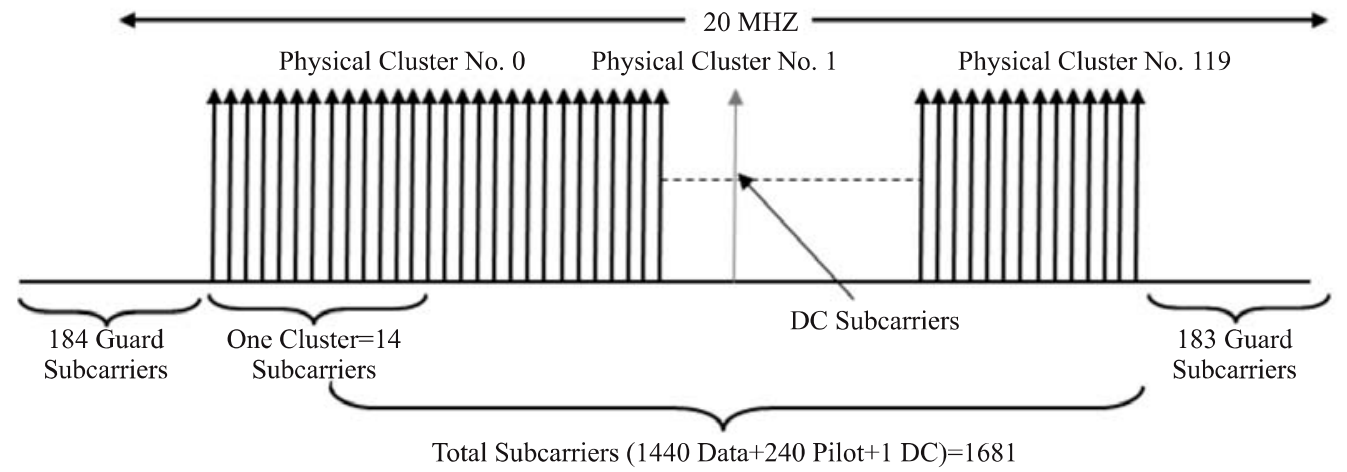

FIG. 4. DOWNLINK PUSC WITH 2048 FFT[3]

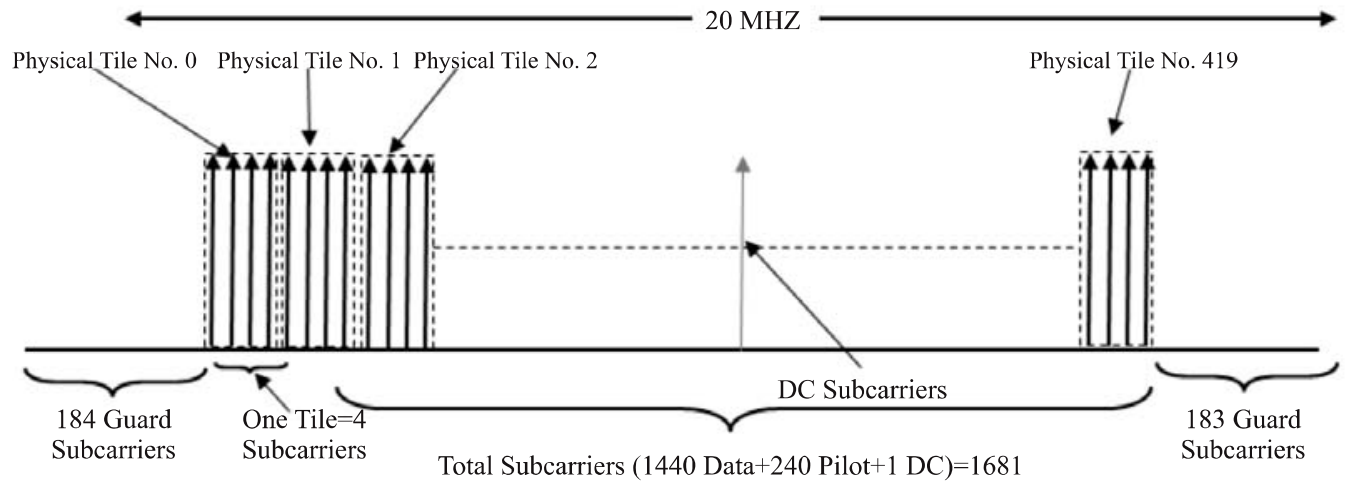

FIG. 5. PUSC UPLINK WITH 2048 FFT [3] 


\section{CONTIGUOUS SUBCARRIER PERMUTATION}

With contiguous subcarrier permutation, sub-channels are composed of contiguous subcarriers and have better channel estimation capabilities [8]. It allows adaptive modulation and coding and Subscriber Station may switch from Distributed-Subcarrier-Permutation to Contiguous-Subcarrier-Permutation by adapting channel conditions. The sub-channel in adjacent subcarrier permutation is composed of bins. A subchannel is constituted of six bins by default but it can vary with respect to slot structure. Four rows of bins form a physical band and group of physical band form logical band. For 2048/1024-FFT, we can get ration of physical bands over logical band simply by dividing maximum number of logical bands by total available physical bands (Fig. 7).

\section{RELATED WORK}

WiMAX was originally designed for Broadband Wireless Access and progressed with time until becoming a candidate technology for the 4G mobile networks. In 90s, IEEE started the working group IEEE 802.16 to create a PMP (Point-to-Multi-Point) air interface alternative to cable and digital subscriber lines.

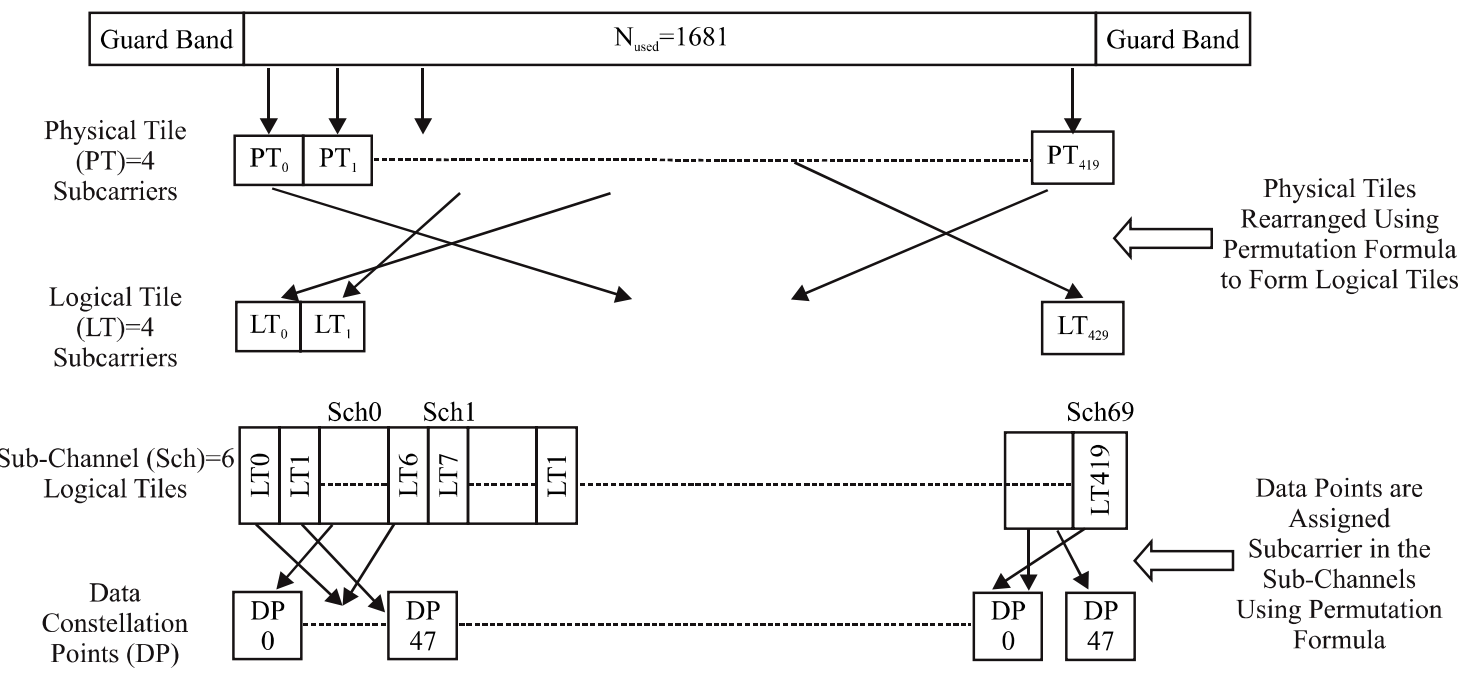

FIG. 6. DATA ALLOCATION TO SUBCARRIERS IN PUSC UL (2048-FFT) [3]

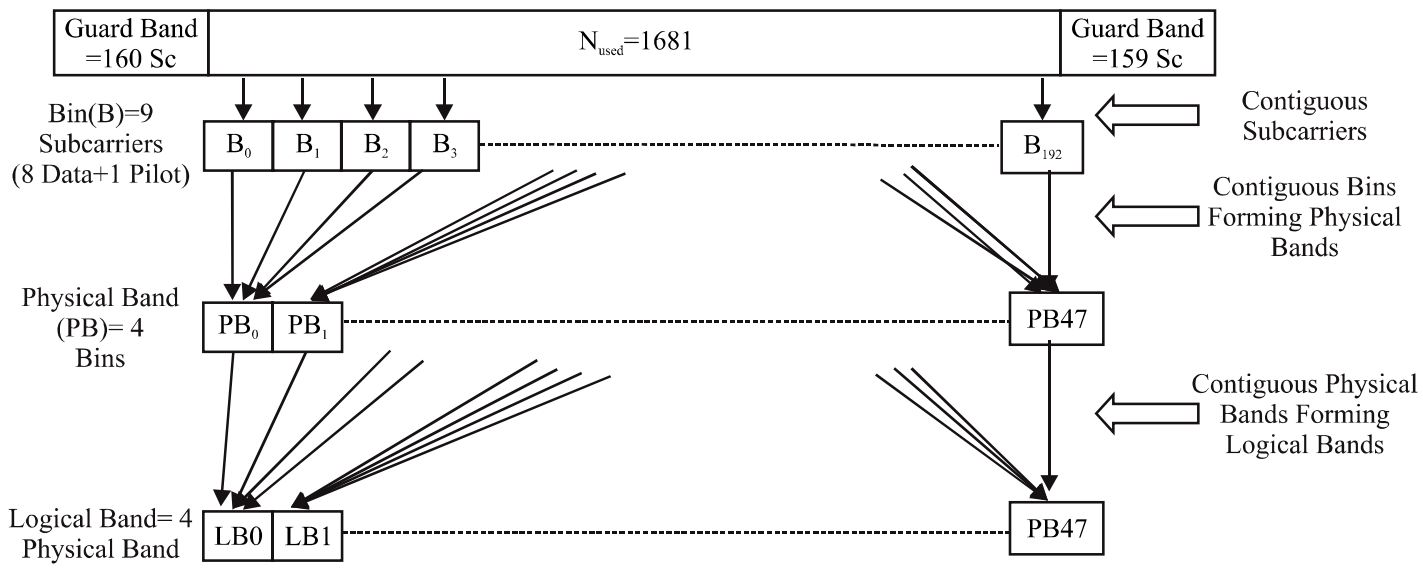

FIG. 7. PHYSICAL AND LOGICAL BANDS IN CONTIGUOUS SUBCARRIER PERMUTATION 
The original standard was modified to produce the IEEE 802.16d standard for fixed applications. IEEE 802.16d uses Orthogonal Frequency-Division Multiplexing (OFDM) as the PHY layer scheme. In 2005, mobility support was incorporated based on SOFDMA (Scalable Orthogonal Frequency-Division Multiple Access), resulting in the amendment 802.16e, also known as Mobile WiMAX [2]. Later, the standard IEEE 802.162009 was released to support both fixed and mobile wireless communications. A complete survey of the historical evolution of the standard up to 2010 can be found in [9]. In 2011, WiMAX evolved to 802.16m [10], which focuses on providing an advanced air interface to fulfill the requirements of IMT-Advanced while maintaining backward compatibility with existing specifications. In August 2012, the IEEE 802.16-2012 [11] was released, consolidating material from IEEE 802.16j2009 for relay-based networks and the amendment 802.16h-2010, which implements coexistence enhancement for license-exempt operation. Such a standard also incorporated the IEEE 802.16m-2011, but excluding the WirelessMAN Advanced Air Interface, which is now specified in the IEEE 802.16.1-2012 [12]. Finally, improvements focused on M2M (Machine-toMachine) applications are examined in amendments 802.16p-2012 [13] and 802.16.1b-2012 [14].

WiMAX PHY profile is provisioned for using Frequency Reuse Factor of one [15]; this results in improved spectral efficacy but result in CCI at cell boundaries. $\mathrm{CCI}$ caused by $\mathrm{FRF}=1$ is required to be measured so that some interference averaging/ minimization techniques can be combined to keep the interference level to some acceptable threshold in wireless environment [16].

The WiMAX physical layer is in charge of multiplexing user and system data together with control signaling in order to ensure a proper utilization of the radio resources.
The WiMAX physical layer specifies how to map and how to allocate those resources as either reference signals or to form various physical channels. WiMAX supports several physical layer modes. Among them, OFDMA is the most appealing given its flexibility and ability to support multiple users at the same time. A very useful review of Physical Layer of Mobile WiMAX System and OFDM is available in [17]. Pedro, et. al. [18] has exploited real time UL and DL concurrent communication and has presented as software defined radio architecture for the real-time implementation PHY Layer for WiMAX transceivers.

\section{SIMULATION SETUP}

For parametric analysis of using different permutation base and its effect on Co-Channel Interference we used OPNET modeler and developed test scenarios. The simulated WiMAX network is a seven cell network where all cells are using same PHY layer profile and same permutation base, initially (Fig. 8). The PHY profile uses Wireless OFDMA with $20 \mathrm{MHz}$ configured for Time Division Duplexing mode. A total of 2048 subcarriers are defied, the Uplink as well as downlink is initially designed using 2048-FFT Partially Utilized Subcarrier.

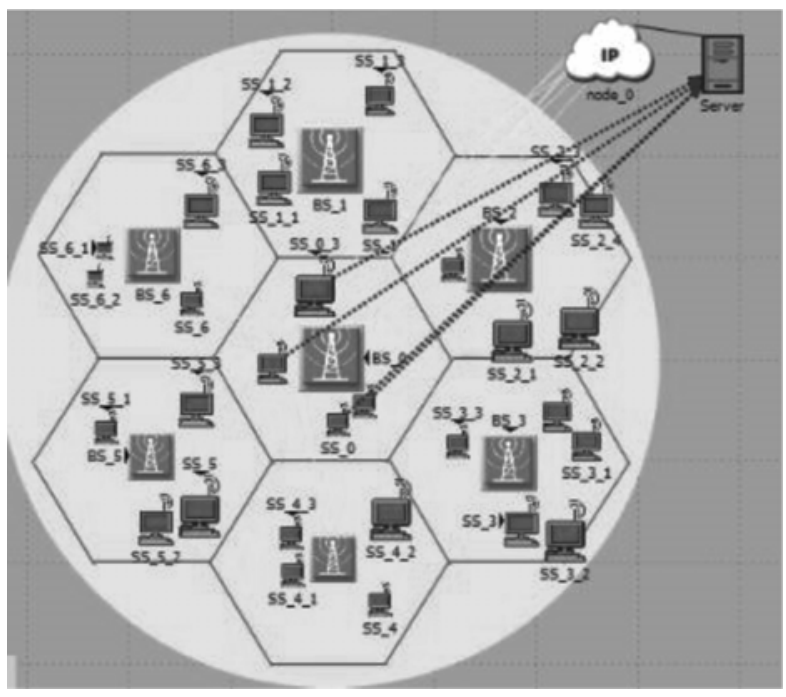

FIG. 8. A SEVEN CELL WIMAX NETWORK CONFIGURED WITH WIMAX PHY PROFILE

Mehran University Research Journal of Engineering \& Technology, Volume 35, No. 4, October, 2016 [p-ISSN: 0254-7821, e-ISSN: 2413-7219] 


\section{SIMULATION RESULTS AND ANALYSIS}

The simulated WiMAX network is initially configured with all the cells in the simulated network use same PHY layer profile and same permutation base. Network statistics are recorded during time interval from 180 and 260 seconds (Fig. 9). It is observed that at this time interval there is increase in WiMAX UL Data burst usage for BS_0 (the

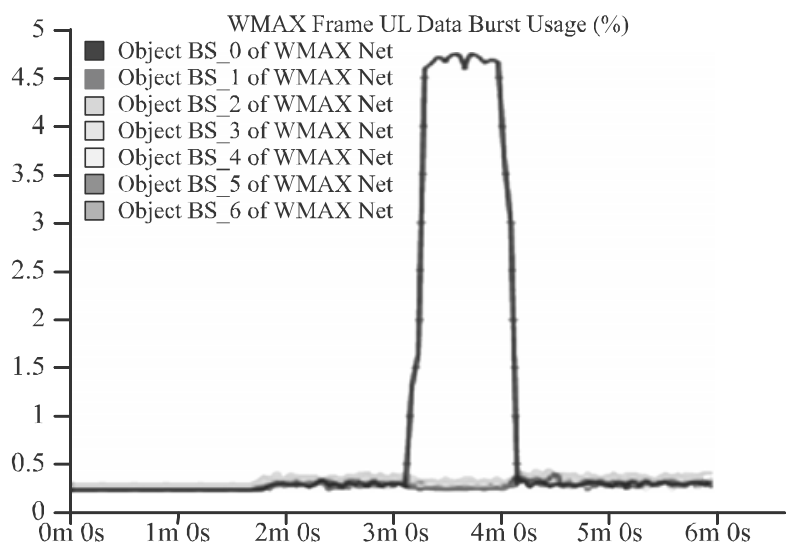

FIG. 9. UL DATA BURST USAGE IN HIGH LOAD CONDITION AT BS_0
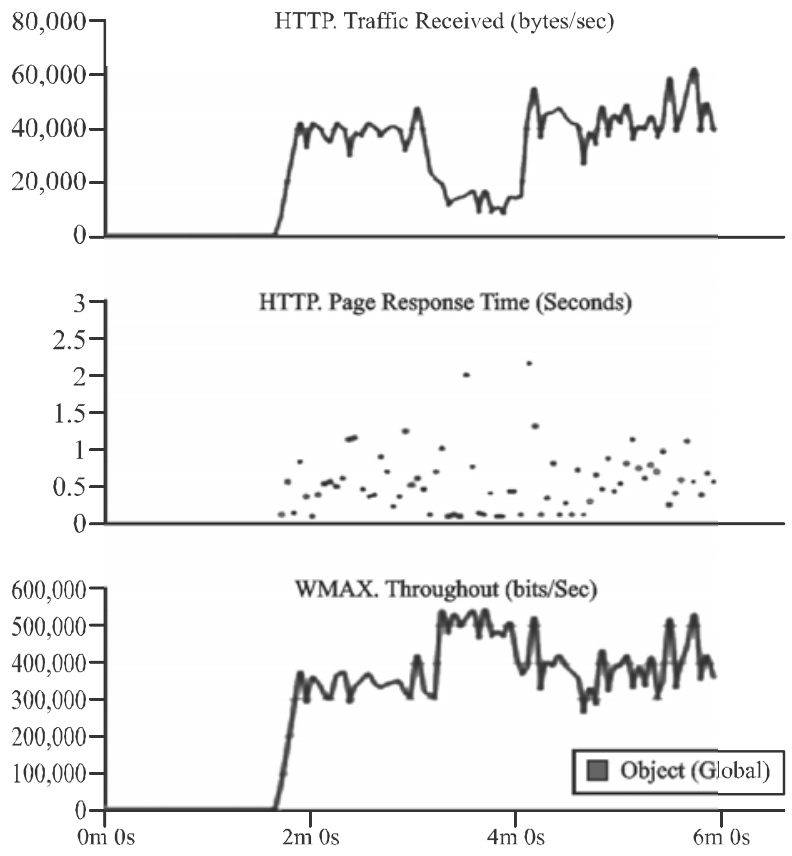

FIG. 10. NETWORK RESPONSE (GLOBAL) IN HIGH LOAD CONDITIONS center cell); this is because of SS (Subscriber Stations) in Cell_0 start transmitting on UL path, sporadically. Network's composite/global response is recorded during this periodic rise in UL traffic and is presented in Fig. 10. It is anticipated that due to high load conditions an increased CCI affected the overall performance of network. From the observations recorded in Fig. 10 it is quite evident that global HTTP received traffic is decreased during the period of increased load in Cell_0 with spontaneous increase in HTTP page response time. With the rise in traffic load the throughput is however increased.

To verify that this global impact on performance metrics, is due to FRF of 1 which resulted in CCI in the network and to further demonstrate the effect of using different permbase on CCI and overall network performance we developed network scenarios using different permutation base value assigned to different cells. Fig. 11 contains BS_0 UL Data burst usage statistics when configured with different permbase. It is noticed that there is no effect on WiMAX Frame UL Data Burst usage when configured with same and different permutation base. This is a supportive observation that the UL burst usage remained unchanged at BS_0 regardless of same or different permutation base used in network.

Fig. 12 presents a comparative analysis of two network scenarios and it is observed that during the high load time i.e. from 180s to 260s the effect of CCI in on overall network behavior is noticeably improved by configuring adjacent cells with different permutation base and thus

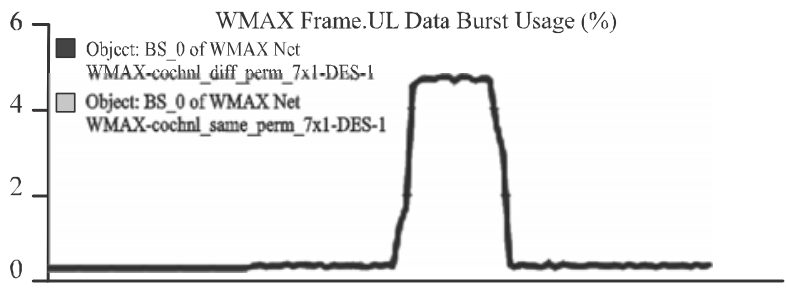

FIG. 11. UL DATA BURST USAGE IN HIGH LOAD CONDITION AT BS_0 WITH SAME AND DIFFERENT PERMBASE AT ADJACENT CELL

Mehran University Research Journal of Engineering \& Technology, Volume 35, No. 4, October, 2016 [p-ISSN: 0254-7821, e-ISSN: 2413-7219] 
usefulness of different permutation base at adjacent cells could be established. Fig. 12 further demonstrate that overall network response is much improved and there are very encouraging results such as improvement in network throughput, HTTP response time is now limited to 1 second which was previously heading to 2 3 seconds. Moreover HTTP traffic sent and received to global node is on higher side which further demonstrates better network performance. Another very important observation is that the retransmission count has noticeably decreased which can be termed as towards improved reliability and dependability of the network.

In order to evaluate the effect of cell sectoring the network is reconfigured with three sectors per each cell; the further configuration of baseline scenario was kept as before. Each sector in cell is configured with different WiMAX PHY profile. However initially all sectors were made to use same permutation base in entire network.
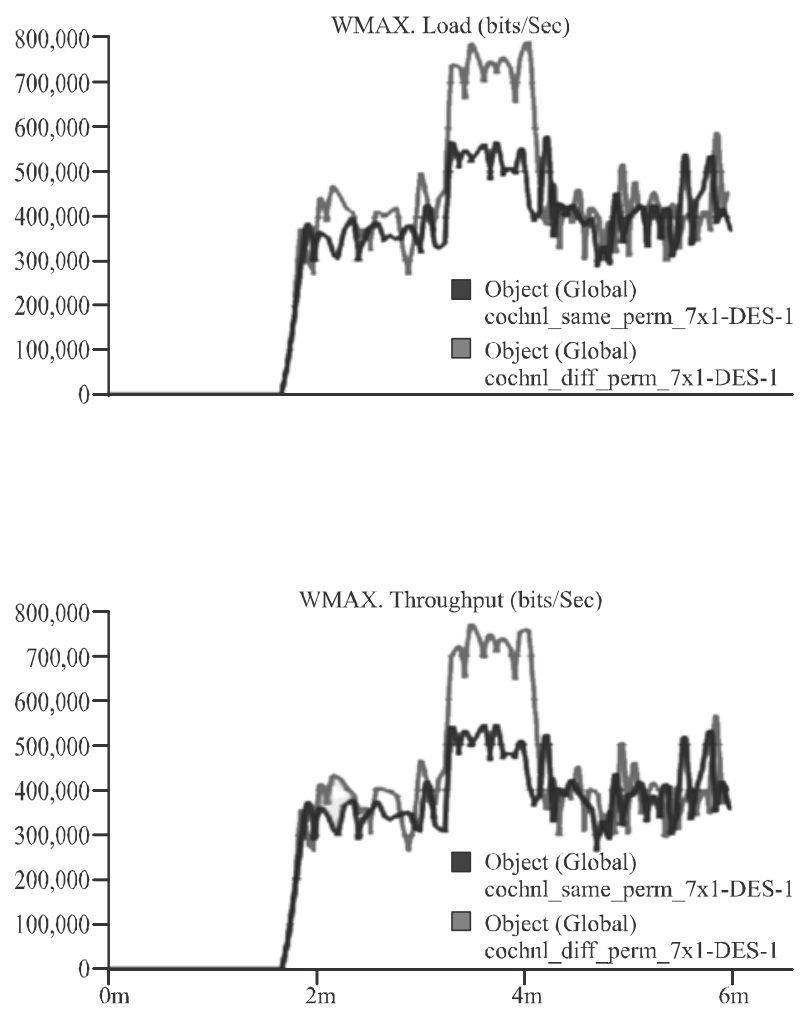

Referring the results in Fig. 13, using cell sectoring technique help improve overall network performance but is found less efficient when compared with technique of using different permutation base in adjacent cells. Using cell sectoring technique may be considered as second choice to improve overall network performance when contrasted the result of two approaches in discussion and evaluation against performance benchmark.

We further extended the parametric analysis and evaluated the effect when two approaches coexist. The results of simulation are grouped in Fig. 14. The comparative analysis of results indicate that such high grade of diversity in network configuration at PHY layer has resulted in improved network statistics and have provided more reliability and better utilization of spectrum.
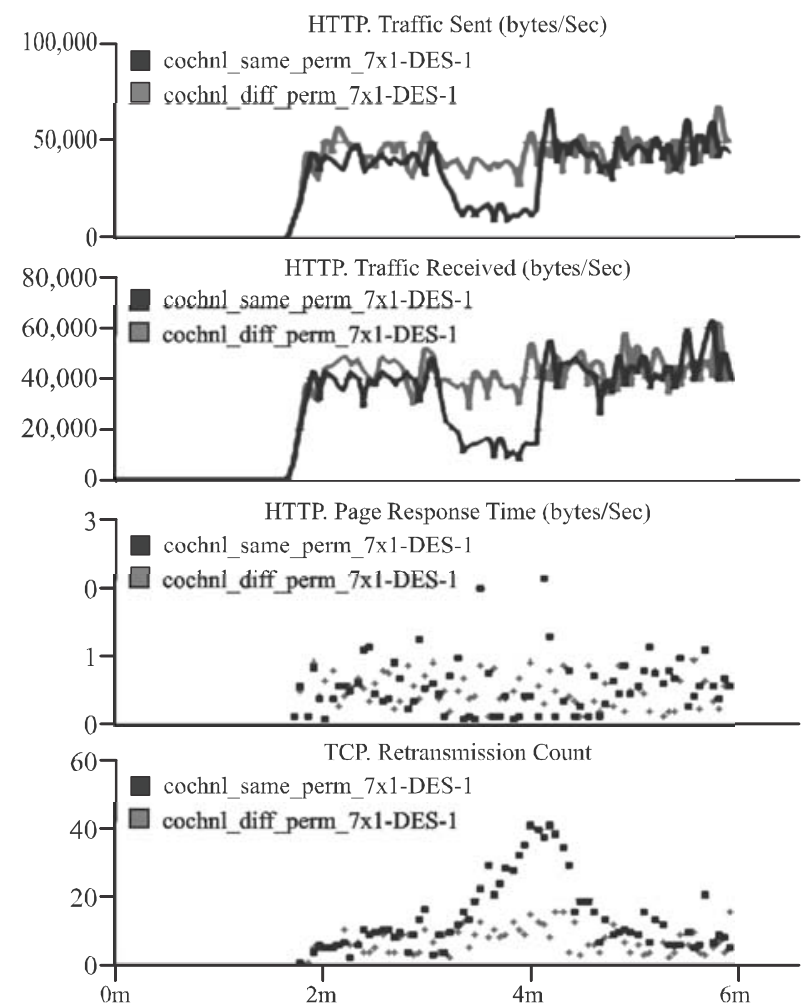

FIG. 12. NETWORK RESPONSE ANALYSIS: SAME/DIFFERENT PERMBASE IN ADJACENT CELLS 


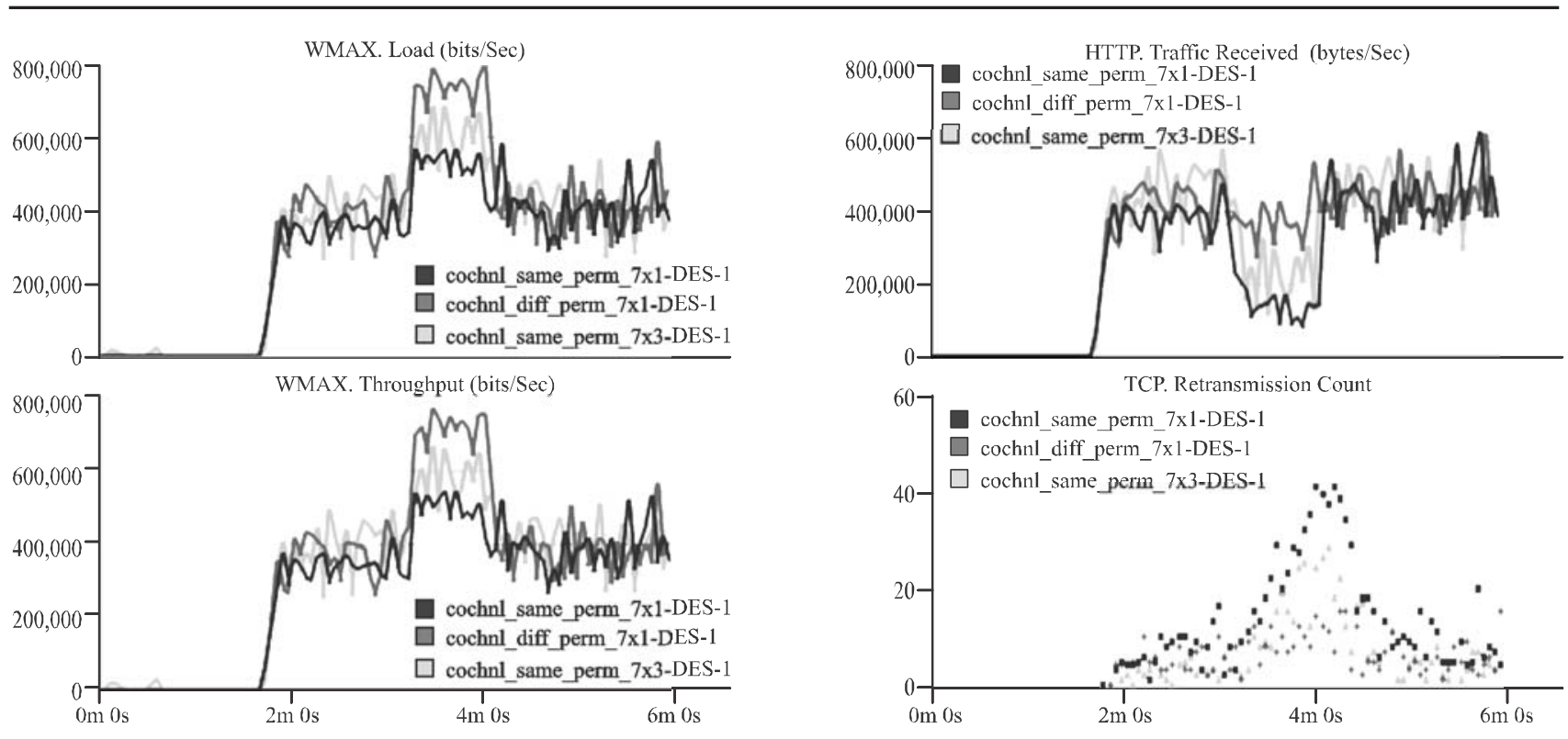

FIG. 13. NETWORK RESPONSE- SAME PERMBASE WITH CELL SECTORING
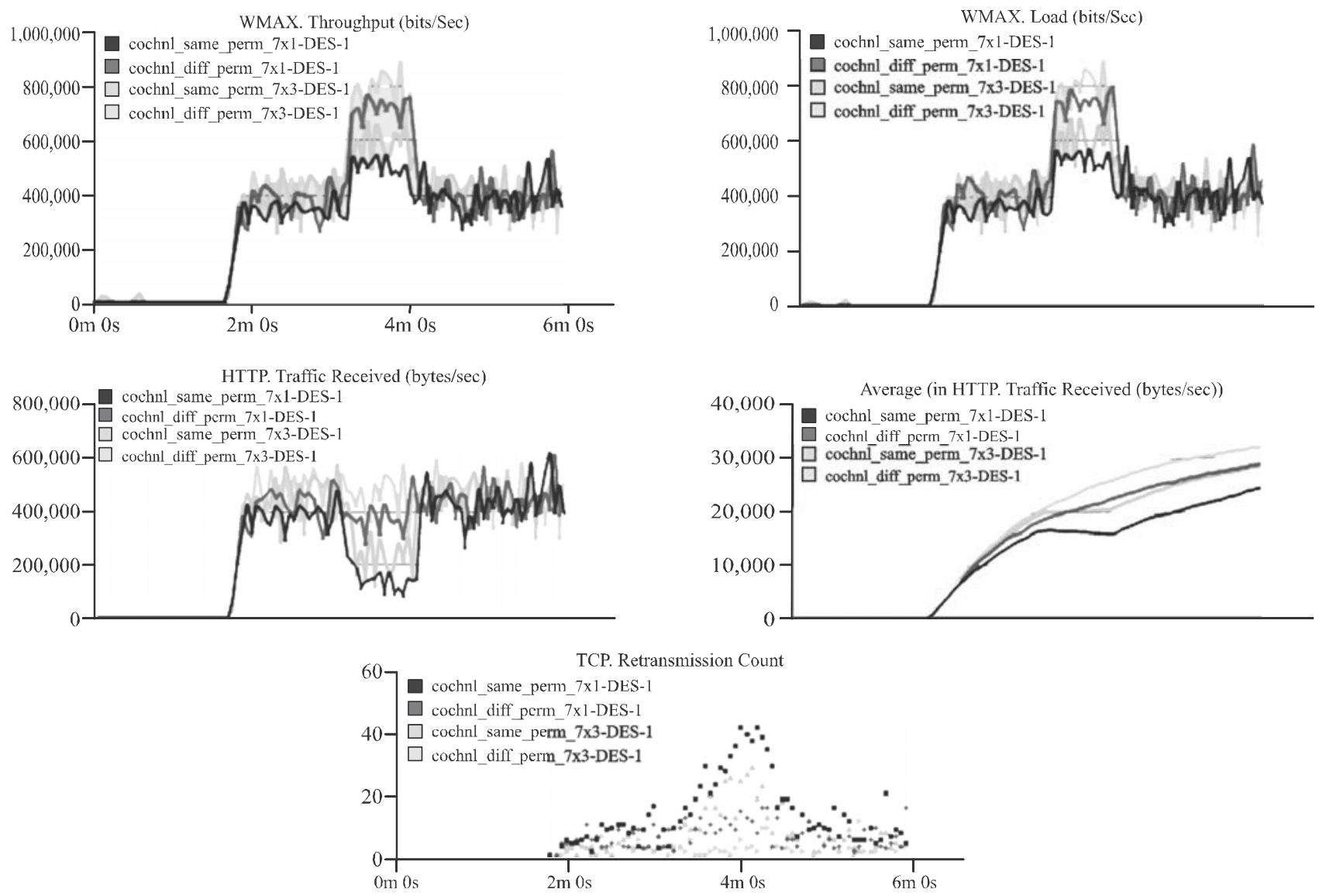

FIG. 14. NETWORK RESPONSE-SAME AND DIFFERENT PERMBASE WITH CELL SECTORING

Mehran University Research Journal of Engineering \& Technology, Volume 35, No. 4, October, 2016 [p-ISSN: 0254-7821, e-ISSN: 2413-7219] 


\section{CONCLUSION}

In this paper we have analyzed the effect of CCI at cell boundaries due to the use of frequency reuse factor of one, and have evaluated the effect of using different permutation base in adjacent cells on CCI. Secondly, we have analyzed the effect of cell sectoring technique on CCI. The parametric review of results using simulated network in OPNET is carried and it is observed that the CCI impact can be lessened by using different permutation base at adjacent cells in a WiMAX network during increased load conditions. More over if same permutation base is used in adjacent cells and sectors are formed it also help decrease CCI impact. Finally the two approaches to mitigate CCI effect are made to coexist in network design and results are compared with previous observations. It is identified that for better spectral efficacy the coexistence of the two approaches has very useful impact on network performance especially it provides noticeable decrease in packet retransmission count and thus provides strong motivation for dependable wireless transport suing WiMAX .

\section{ACKNOWLEDGEMENT}

We acknowledge the Higher Education Commission of Pakistan for its support under National Research Grants Program for Universities.

\section{REFERENCES}

[1] Lawton, G., "What Lies Ahead for Cellular Technology?”, Article in Industry Trends, IEEE Computer Society, pp. 14-17, June, 2005.

[2] IEEE Standard 802.16e, "Part-16: Air Interface for Fixed and Mobile Broadband Wireless Access Systems”, December, 2005.

[3]

IEEE 802.16-2004, IEEE Standard for Local and Metropolitan "Area 200 Networks- Part-16: Air Interface for Fixed Broadband Wireless Access Systems”, October, 2004.
[4] Kulkarni, G., Adlakha, S., and Srivastava, M., "Subcarrier Allocation and Bit Loading Algorithms for OFDMABased Wireless Networks”, IEEE Transactions on Mobile Computing, Volume 4, No. 6, pp. 652-662, December, 2005.

[5] IEEE Standard. 802.16e, IEEE Standard for Local and Metropolitan Area Networks, "Part-16: Air Interface for Fixed and Mobile Broadband Wireless Access Systems, Amendment 2: Physical and Medium Access Control Layers for Combined Fixed and Mobile Operation in Licensed Bands and Corrigendum 1", May, 2005.

[6] Krishna, R., and Raj, J., "WiMAX System Evaluation Methodology”, WiMAX Forum, January, 2007.

[7] Yaghoobi, H., "Scalable OFDMA Physical Layer in IEEE 802.16 Wireless MAN”, Intel Technical Journal, Volume 8, No. 3, pp. 201-212, 2004.

Masoud, O., "Introduction to WiMAX", OPNETWORK, Emerging Access Technologies Research Group SPRINT and NEXTEL, August, 2006.

[9] Pareit, D., Lannoo, B., Moerman, I., Demeester, P., "The History of WiMAX: A Complete Survey of the Evolution in Certification and Standardization for IEEE 802.16 and WiMAX”, IEEE Communications Surveys Tutorials, Volume 14, No. 4, pp. 1183-1211, 2012 [doi:10.1109/SURV.2011.091511.00129]

[10] IEEE Standard for Local and Metropolitan Area Networks "Part-16: Air Interface for Broadband Wireless Access Systems Amendment 3: Advanced Air Interface”, IEEE Standard 802.16m-2011 (Amendment to IEEE Sandatd 802.16-2009, [doi:10.1109/IEEESTD.2011.5765736], 2011.

[11] IEEE Standard "Air Interface for Broadband Wireless Access Systems” IEEE Standard 802.16-2012, Revision of IEEE Standard 802.16-2009, pp. 1-2542, 2012 [dio: 10.1109/IEEESTD.2012.6272299]

[12] IEEE Standard "WirelessMAN-Advanced Air Interface for Broadband Wireless Access Systems”, IEEE Standard 802.16.1-2012, pp. 1-1090, 2012 [doi:10.1109/ IEEESTD.2012.6297413] 
[13] IEEE Standard, "Air Interface for Broadband Wireless Access Systems-Amendment 1: Enhancements to Support Machine-to-Machine Applications”, IEEE Standard 802.16p-2012, Amendment to IEEE Standard 802.16-2012, pp. 1-82, 2012 [doi:10.1109/ IEEESTD.2012.6327306]

[14] IEEE Standardm Wireless MAN-Advanced Air Interface for Broadband Wireless Access Systems Amendment 1: Enhancements to Support Machine-to-Machine Applications”, IEEE Standard 802.16.1b-2012, Amendment to IEEE Standard 802.16.1, pp..1-126, 2012 [doi:10.1109/IEEESTD.2012.6328224]

[15] Maqbool, M., Coupechoux, M., and Godlewski, P., "Comparison of Various Frequency Reuse Patterns for WiMAX Networks with Adaptive Beamforming”, Proceedings of IEEE VTC Spring, pp. 2582-2586, May, 2008.
[16] Viering, I., Klein, A., Ivrlac, M., Castaneda, M., and Nossek, J., “On Uplink Inter-Cell Interference in a Cellular System”, Proceedings of IEEE International Conference on Communications, pp. 2095-2100, June, 2006.

[17] Kavita, D., and Hemant, D., "A Review of Physical Layer of Mobile WiMAX System and OFDM", International Journal of Science and Research, Paper ID: SUB15325, Volume 4, No. 1, pp. 1518-1521, 2015.

[18] Pedro S., "A Real-Time Implementation of the Mobile WiMAX ARQ and Physical Layer”, International Journal of Signal Processing Systems, Volume 78, pp. 283-297, 2015. 